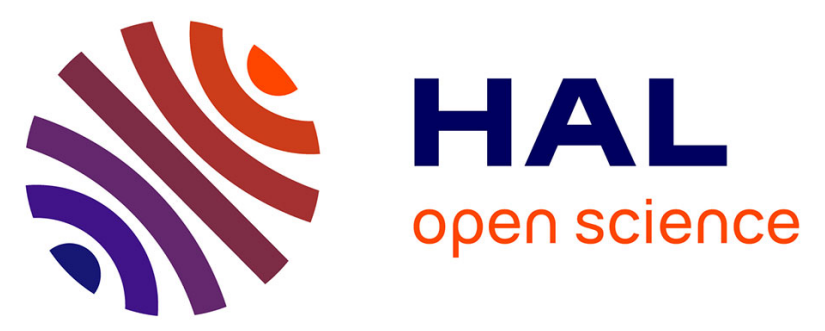

\title{
How to build an average model when samples are variably incomplete? Application to fossil data
}

Jean Dumoncel, Gérard Subsol, Stanley Durrleman, Jean Pierre Jessel, Amélie Beaudet, José Braga

\section{- To cite this version:}

Jean Dumoncel, Gérard Subsol, Stanley Durrleman, Jean Pierre Jessel, Amélie Beaudet, et al.. How to build an average model when samples are variably incomplete? Application to fossil data. CVPRW: Computer Vision and Pattern Recognition Workshops, Jun 2016, Las Vegas, United States. pp.541548, 10.1109/CVPRW.2016.74 . lirmm-01381310

\section{HAL Id: lirmm-01381310 \\ https://hal-lirmm.ccsd.cnrs.fr/lirmm-01381310}

Submitted on 14 Oct 2016

HAL is a multi-disciplinary open access archive for the deposit and dissemination of scientific research documents, whether they are published or not. The documents may come from teaching and research institutions in France or abroad, or from public or private research centers.
L'archive ouverte pluridisciplinaire HAL, est destinée au dépôt et à la diffusion de documents scientifiques de niveau recherche, publiés ou non, émanant des établissements d'enseignement et de recherche français ou étrangers, des laboratoires publics ou privés. 


\section{How to build an average model when samples are variably incomplete? Application to fossil data}

\author{
Jean Dumoncel \\ UMR AMIS \\ CNRS-University of Toulouse, France \\ Stanley Durrleman \\ UMR ICM \\ INRIA-University of Paris 06, France \\ Amélie Beaudet \\ Department of Anatomy \\ University of Pretoria, South Africa
}

\author{
Gérard Subsol \\ UMR LIRMM \\ CNRS-University of Montpellier II, France \\ Jean-Pierre Jessel \\ UMR IRIT \\ CNRS-University of Toulouse, France \\ José Braga \\ UMR AMIS \\ CNRS-University of Toulouse, France
}

\begin{abstract}
In paleontology, incomplete samples with small or large missing parts are frequently encountered. For example, dental crowns, which are widely studied in paleontology because of their potential interest in taxonomic and phylogenetic analyses, are nearly systematically affected by a variable degree of wear that alters considerably their shape. It is then difficult to compute a significant reference surface model based on classical methods which are used to build atlases from set of samples. In this paper, we present a general approach to deal with the problem of estimating an average model from a set of incomplete samples. Our method is based on a state-of-the-art non-rigid surface registration algorithm. In a first step, we detect missing parts which allows one to focus only on the common parts to get an accurate registration result. In a second step, we try to build average model of the missing parts by using information which is available in a subset of the samples. We specifically apply our method on teeth, and more precisely on the surface in between dentine and enamel tissues (EDJ). We investigate the robustness and accuracy properties of the methods on a set of artificial samples representing a high degree of incompleteness. We compare the reconstructed complete shape to a ground-truth dataset. We then show some results on real data.
\end{abstract}

\section{Introduction}

The recent developments in 3D imaging allow finer analysis, particularly in the field of paleontology [28]. The study of the morphology and morphometry of the fossil anatomical structures leads to find some relationships between extant and fossil taxa. More precisely, the characterisation of shape features represented in each group together with the identification of both similarities and differences between samples is a prerequisite for understanding the past and current biodiversity. Moreover, the access to large databases allows to compare anatomical structures and to potentially answer questions about fossil animals dealing with the taxonomy, which consist in naming and classifying organisms. Unfortunately, these samples are often altered by biological modifications (e.g. wear of dental crowns) or damaged by post-mortem taphonomic processes (e.g. breakages, deformations) and thus present missing data [19, 22, 26]. To solve this problem, we have first to develop new tools to quantify at best the modern and fossil variability. Then we have to deal with the problem of the distortion of statistical analyses due to missing data. In conclusion, once we have a set of surfaces, how can we perform comparative analyses of their shape with incomplete samples?

The so-called "geometric morphometrics" propose a procedure to study comparative anatomy [6, 18]. These methods are based on corresponding points which are manually defined, also called landmarks. The variations of shapes are then quantified by using the cartesian coordinates of anatomical landmarks. In a first step, a "generalised Procrustes analysis" [16] is performed to align sets of landmarks between them by using a procedure of least squares based on translations, rotations and scaling. Procrustes coordinates describe then every sample in the socalled Kendall shape space [21].

A recent alternative of these methods is to automatically 
determine correspondences between the anatomical structures. We can use many methods of 3D rigid and non rigid registrations for the automatic detection of such correspondences. The application to fossil is often based on the "iterative closest point" [5, 27] algorithm but the alignments are not effective when large distortions or discontinuities are observed among sample surfaces. Algorithms of automatic alignment of shapes were also developed, but these methods are not really widespread in the field of paleontology or of medical imaging [1, 7, 25].

In this paper, we use a registration method based on a mathematical model which computes a continuous diffeomorphic evolution of the shapes from discrete set of observations [11, 13]. This flow of diffeomorphism allows to compute parameters for shape analysis. Through this method, fields of deformation computed in a volume containing surfaces are produced. In this form, the deformations can be used at the same time in the statistical models.

Two main categories of methods for handling incomplete samples are usually recognised: deletion of incomplete samples, or reconstruction of missing parts [10]. In this paper, we propose an additional strategy. We examine how average shape can be combined with concepts of diffeomorphism to obtain consistent descriptions even in the case of missing part, by following the successive steps; (i) first we deform a template to each sample, (ii) secondly we compute a average shape and (iii) finally we study the influence of missing data in relation to the problematics of fossil data with an application to paleontology.

\section{Framework to estimate an average model}

\subsection{Atlas and average model}

Anatomical atlases are becoming widespread in the anatomical studies, whether it is to establish a diagnosis, or follow the evolution of a disease [24] but also in paleontology to discriminate taxonomic groups (e.g., species, genera). Atlas based on morphometric tools, that integrates the notion of variability, is more and more used [9, 14].

There are several definitions for the building of an average shape from anatomical structures depending on the nature of the data analysed [17]. In [23], the authors describe a method based on a search of landmarks and of characteristic lines in surfaces. The notion of average shape is bound to that of statistical model, indeed, the average shape is generated with the aim of exploring the variability of a set of shapes to be subsequently used either for the segmentation, or for the statistical analyses of populations.

In this paper, the average shape is based on computing the deformations between a reference shape called template and all the samples. Of course, these deformations are sensitive to all the geometric distortions of the samples, in particular the missing parts. The difficulty is then to take into account the missing parts which are not known a priori. Here we use the registration framework and the atlas building method described in [13]. However, we use a different definition of the average shape. The aim is to begin with a given template $T_{0}$ and a set of samples $X_{1} \ldots X_{N}$, and then to compute the deformations $\phi_{i}$ between $T_{0}$ and each sample $X_{i}$ and to use all the $\phi_{i}$ to compute an average deformation which will be applied to $T_{0}$ in order to find the average shape $\bar{T}$. In the original method, at each step of the registration process, the average shape is updated and refined and becomes a new template. In this work, we choose to use a straightforward procedure: we compute the average shape once, at the end of the registration process by averaging the resulting deformations $\phi_{i}$. This will give a less accurate average shape but the problem is that we do not know a priori where are the missing parts in the samples and so, we do not want the template to be distorted by the influence of the missing parts during registration.

\subsection{Registration methodology}

For non-rigid surface registration, we are going to use the Deformetrica software (http://www.deformetrica.org) [12]. Let a set of 3D meshes $X_{i}$. We assume that all the $X_{i}$ have been previously aligned, i.e. the difference of position, orientation and scaling were removed by performing a rigid and scale registration. Each $X_{i}$ can be defined by:

$$
\left\{\begin{array}{l}
X_{i}=T_{0}^{i}+\epsilon_{i} \\
T_{0}^{i}=\phi_{i}\left(T_{0}\right)
\end{array}\right.
$$

Where $\phi_{i}$ is the deformation (in our case a diffeomorphic one) between a template $T_{0}$ and $X_{i}, \epsilon_{i}$ represents the residue between the deformed template $T_{0}^{i}$ and $X_{i}$.

In Deformetrica, template-to-sample deformations $\phi_{i}$ are estimated by minimising the criterion:

$$
E\left(T_{0}, \phi_{i}\right)=D\left(\phi_{i}\left(T_{0}\right), X_{i}\right)+\operatorname{Reg}\left(\phi_{i}\right)
$$

Where $D$ is the squared distance between so-called currents and $\operatorname{Reg}\left(\phi_{i}\right)$ a measure of regularity of the deformations. The diffeomorphic deformation $\phi_{i}$ is defined as a set of control points $\mathrm{CP}$, which positions are constant over iterations, and a set of parameters $\alpha_{i}$ called "momenta". In our experiments, the control points are distributed on a regular 3D grid where the distance between two control points is $1 \mathrm{~mm}$. For the other parameters of the registration algorithm, we used "standard" values advocated by the authors of Deformetrica : 1 for the width of the gaussian kernel (to be compared to the dimensions of a tooth which are about $15 \times 10 \times 10 \mathrm{~mm}$ ) and 0.1 for the weight of the data term to enforce a good matching accuracy.

In the following, we present three different workflows to study the influence of missing parts in next sub-sections 2.3. 2.4 and 2.5 (see also Figure 1). 


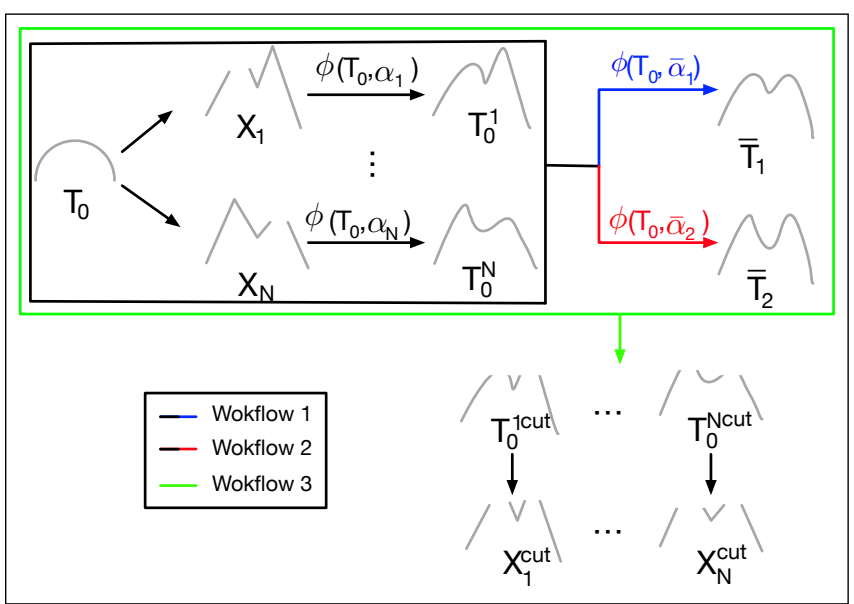

Figure 1: Schematic view of the three workflows showing the different strategies for handling incomplete samples.

\subsection{Average shape without taking into account missing parts}

As the set of momenta $\alpha_{i}$ completely describes the deformation $\phi_{i}$, we will perform the average of the $\alpha_{i}$ to find the average deformation. Applied to $T_{0}$, it will result in the average shape $\bar{T}_{1}$. This will define workflow 1 which we will use as a reference to analyse the influence of missing parts.

\footnotetext{
Workflow 1:

1. For each sample $X_{i}$, compute the deformation $\phi_{i}$ defined by $\alpha_{i}$.

2. Compute the average of the set of momenta: $\bar{\alpha}_{1}=$ $\frac{\sum_{i} \alpha_{i}}{N}$.

3. Compute the deformation of the template $T_{0}$ according to the average of the set of momenta $\bar{\alpha}_{1}$. The result is the average shape $\bar{T}_{1}$.
}

\subsection{Average shape with detection of missing parts}

Because the deformations $\phi_{i}$ do not take into account the missing parts and extrapolate absent correspondences, we propose a second workflow to discard deformation parameters around the missing parts. This consists in first detecting the missing parts and then to not use the subset of momenta which are associated to the control points in the neighbourhood of the detected parts. This will allow us to get an average model which will be less distorted by the absence of some parts.

\section{Workflow 2:}

1. For each sample $X_{i}$, compute the deformation $\phi_{i}$ defined by $\alpha_{i}$.

2. We have the deformed template $T_{0}^{i}=\phi_{i}\left(T_{0}\right)$ :

2.1. Find vertices $V_{j}$ of $T_{0}^{i}$ which have "no correspondent" on the target sample $X_{i}$. These vertices are defined as having a closest point on $X_{i}$ which is distant of more than a given distance $D_{v}$.

2.2. Find control points $C P_{c}$ close to $V_{j}$. A control point $C P_{k}$ is considered close if $D\left(C P_{k}, V_{j}\right)<D_{c p}$. Let us define $\alpha_{c}$ the set of associated momenta to $C P_{c}$.

2.3. $\alpha_{i}^{\prime}=\alpha_{i} \backslash \alpha_{c}$ is the set of active momenta, i.e. the ones which will be used in the final average for the sample $i$.

3. Compute the average of the set of momenta: $\bar{\alpha}_{2}=$ $\sum_{i} \alpha_{i}^{\prime}$ (i.e. for each set of momenta, we take into account only the active values that we divide by the number of active momenta).

4. $\bar{T}_{2}=\phi\left(T_{0}, \bar{\alpha}_{2}\right)$ is the new average shape which takes into account the missing parts.

\subsection{Average shape based only on common parts}

We present a third workflow to keep only the parts which are common to all the samples. This is a way to detect the most reliable parts, which are present in all the samples. In some cases, we want to use only this information to draw some conclusions about the shape.

Workflow 3:

1. Apply workflow 1 . We get $\bar{T}_{1}$.

2. For each sample $X_{i}$.

2.1. We have the deformed template $T_{0}^{i}=\phi_{i}\left(T_{0}\right)$.

2.2. Find the vertices $V_{j}$ from $T_{0}^{i}$ which have "no correspondent" on the target sample $X_{i}$ based on $D_{v}$.

2.3. Delete $V_{j}$ (which are in different geometrical positions) in $T_{0}^{i}$ and $\bar{T}_{1}$.

3. We obtain $T_{0}^{i, c u t}$ and $\bar{T}_{1}^{c u t}$.

4. We can then use $T_{0}^{i, c u t}$ to cut the sample $X_{i}$ to keep only the vertices which are common to all the samples by:

4.1. Find vertices $V_{k}$ from $X_{i}$ which have "no correspondent" on the cut deformed template $T_{0}^{i, c u t}$. These vertices are defined as having a closest point on $T_{0}^{i, c u t}$ which is distant of more than a given distance $D_{v}$.

4.2. Delete $V_{k}$ in $X_{i}$. We obtain $X_{i}^{c u t}$.

4.3. We can then use $\left(\bar{T}_{1}^{c u t}, X_{1}^{\text {cut }} \ldots X_{N}^{c u t}\right)$ for a new registration process. 


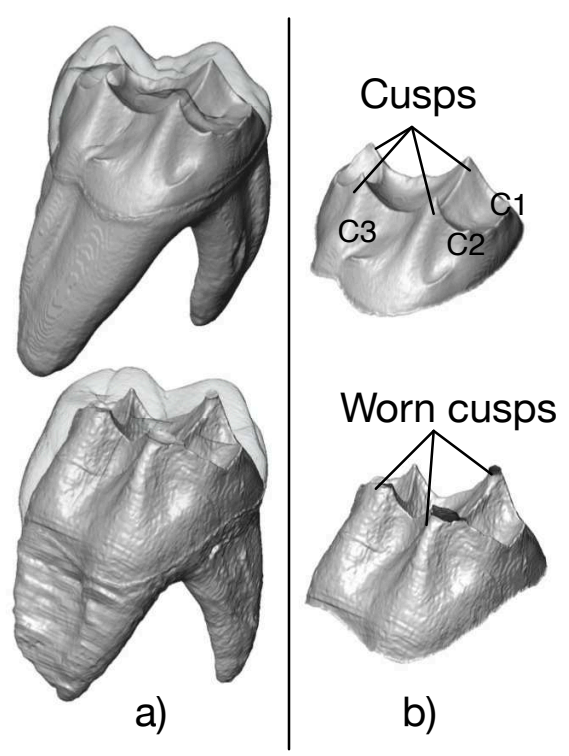

Figure 2: a) Segmentation of dental tissues from two upper third molars. Enamel and dentine are rendered in semitransparency and light grey respectively. b) The extracted enamel-dentine surfaces.

\section{Experiments and results}

Our test cases concern the enamel-dentine junction (EDJ) surface morphology (Figure 2). Teeth are largely studied in paleontology because their morphology is suggested to be informative for discriminating fossil species [8]. The interface between the dentine and the enamel tissues is a surface relatively protected from the outside environment. Dental features extracted from EDJ surfaces vary in shapes and sizes, notably by the conformation of cusps and ridges. However, research have to face the problem of enamel and dentine loss due to dental wear [4, 20] which affect the morphology of the dentine horns. For example, when some cusps are worn (Figure 2), a virtual reconstruction can be made but teeth that showed significant missing areas are excluded from studies.

Some of the EDJs included in our work have been studied in previous publications [2, 3]. All the teeth are upper third molars. Among the four cusps visible on the EDJ, we selected three of them abbreviated C1, C2 and C3 (Figure 2). We choose to study only these three cusps, because the fourth cusp was rarely damaged in our sample. We used four sets for our experiments in order to test the impact of missing data. The first set $\mathrm{S} 1$ is composed of thirteen complete surfaces. The cusps of some samples selected among the S1 sample were cut and included in the second set S2 (Table 1). The same cusps represented in the third set S3 were cut based on the frequencies and percentages observed

\begin{tabular}{|c|c|c|c|c|c|c|}
\hline & \multicolumn{3}{|c|}{ S2 } & \multicolumn{3}{|c|}{ S3 } \\
\hline & $\mathrm{C} 1$ & $\mathrm{C} 2$ & C3 & C1 & $\mathrm{C} 2$ & C3 \\
\hline$X_{1}$ & $100 \%$ & $100 \%$ & $100 \%$ & $60 \%$ & $80 \%$ & 50 \\
\hline$X_{2}$ & - & $100 \%$ & - & $20 \%$ & - & - \\
\hline$X_{3}$ & - & $100 \%$ & $100 \%$ & - & $50 \%$ & $30 \%$ \\
\hline$X_{4}$ & - & $100 \%$ & $100 \%$ & - & $40 \%$ & $10 \%$ \\
\hline$X_{5}$ & - & $100 \%$ & $100 \%$ & - & $60 \%$ & $60 \%$ \\
\hline$X_{6}$ & - & $100 \%$ & $100 \%$ & - & $40 \%$ & $30 \%$ \\
\hline$X_{7}$ & - & $100 \%$ & $100 \%$ & - & $70 \%$ & $10 \%$ \\
\hline$X_{8}$ & $100 \%$ & $100 \%$ & - & $80 \%$ & $50 \%$ & - \\
\hline$X_{9}$ & $100 \%$ & $100 \%$ & $100 \%$ & $70 \%$ & $80 \%$ & $90 \%$ \\
\hline$X_{10}$ & $100 \%$ & $100 \%$ & - & $10 \%$ & $20 \%$ & - \\
\hline$X_{11}$ & $100 \%$ & $100 \%$ & - & $30 \%$ & $60 \%$ & - \\
\hline$X_{12}$ & $100 \%$ & - & - & $20 \%$ & - & - \\
\hline$X_{13}$ & $100 \%$ & $100 \%$ & - & $10 \%$ & $20 \%$ & - \\
\hline
\end{tabular}

Table 1: Cut cusps in the sample - Percentages are given approximately. $100 \%$ indicates we cut the whole cusp, i.e. we cut the EDJ at the basis of the cusp.

\begin{tabular}{|c|ccc|}
\hline & & $\mathrm{S} 4$ & \\
& $\mathrm{C} 1$ & $\mathrm{C} 2$ & $\mathrm{C} 3$ \\
\hline$X_{1}$ & $50 \%$ & $100 \%$ & - \\
$X_{2}$ & $10 \%$ & $70 \%$ & $10 \%$ \\
$X_{3}$ & - & $10 \%$ & $30 \%$ \\
$X_{4}$ & $10 \%$ & $90 \%$ & - \\
$X_{5}$ & $50 \%$ & $90 \%$ & $10 \%$ \\
$X_{6}$ & - & $40 \%$ & - \\
$X_{7}$ & - & $90 \%$ & $10 \%$ \\
\hline
\end{tabular}

Table 2: Visual estimation of the percentage of missing cusps per sample.

on real samples (Table 1). The last set S4 is composed of seven original teeth affected by wear. Accordingly, S2, S3 and S4 do not contain any complete sample (Table 2). Each surface of S1 (resp. S4) was aligned in position, orientation and scale with respect to a reference surface chosen randomly in the same set by using the "Align Surface" module available in the Avizo v8.0 software (which is based on an ICP method). Then we used the aligned surfaces of S1 to generate $\mathrm{S} 2$ and $\mathrm{S} 3$.

To analyse the ability of the method to reconstruct the average shape, we compute the average shapes for the four sets (Figure 3) following the first workflow for S1 and the first and the second workflow for S2, S3 and S4. The template used is a half-sphere aligned with the reference using the "Align Surface" module. Because the deformed templates do not match perfectly the observations, a lower value of $D_{v}$ could lead to interpret inaccurate matching as missing parts while a higher value fails to detect missing parts. The parameter $D_{C P}$ could also influence the shape of the reconstructing average shape. After doing some tests, we chose a distance of $D_{v}=0.2 \mathrm{~mm}$ for the selection of the vertices, and a distance of $D_{C P}=1.5 \mathrm{~mm}$ for the selection of the control points.

The reconstruction of the average shape using the workflow 1 on S2 and S3 clearly shows the influence of missing 

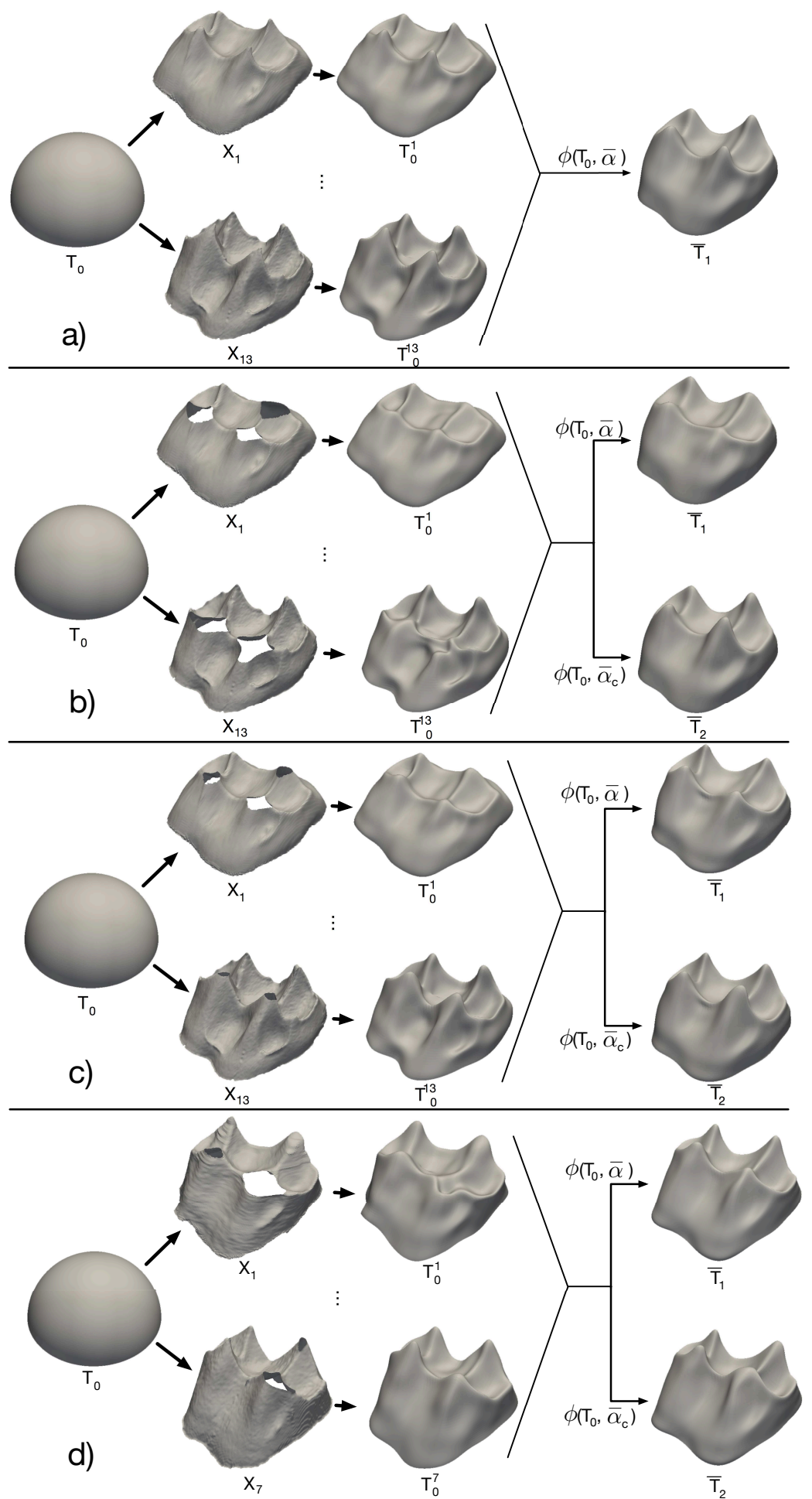

Figure 3: Results of the deformation process performed from the template $T_{0}$ to the sample $X_{i}$ for the four sets $\mathrm{S} 1$ (a; entire teeth), S2 (b; manual cut of S1 with high incomplete parts), S3 (c; manual cut of S1 with realistic incomplete parts) and S4 (d; real teeth. $\bar{T}_{1}$ (resp. $\bar{T}_{2}$ ) is obtained by following workflow 1 (resp. workflow 2 ). 


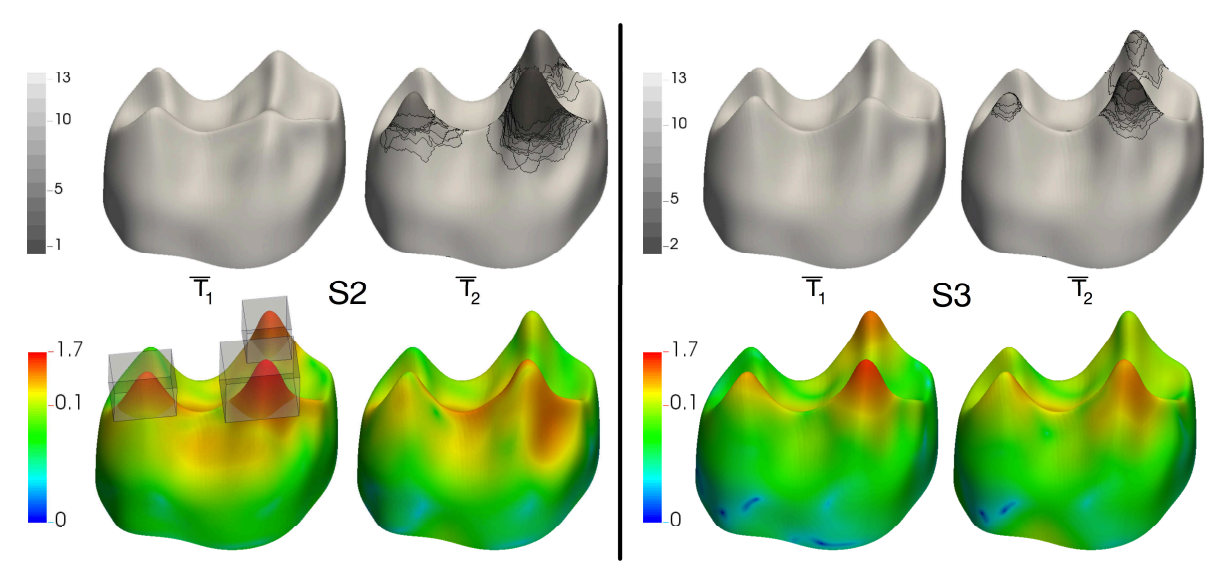

Figure 4: Top; Number of samples used to compute the average shape (left; S2, right; S3) rendered by a pseudo-color scale ranging from black (lowest values) to grey (highest values). Bottom; distances map between the average shape for S1 and the average shape for S2 (left) and S3 (right) using a log blue-green-red colormap. Bottom left; transparent bounding boxes used for the comparison of the cusps.

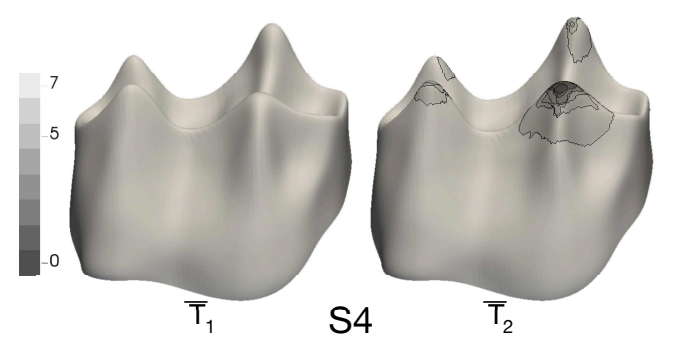

Figure 5: Top; Number of samples used to compute the average shape for $\mathrm{S} 4$ rendered by a pseudo-color scale ranging from black (lowest values) to grey (highest values).

data in the shape of the average shape. For example, in $\bar{T}_{1}$, the cusp C 3 is flattened but in $\bar{T}_{2}$, the same cusp is more prominent. In Figure 3, we show the template-to-sample for each dataset. For S1, the deformed templates show the accuracy of the registration. The deformed templates of S2 show the extrapolation made by the deformation algorithm when some parts are significantly missing. However this artifact can also influence the deformation for smaller missing part as shown for the result of S3. Figure 3 also shows the average shapes for the four sets following the first workflow for S1 and the first and second workflow for S2, S3 and S4.

We further analysed the ability of the method to reconstruct the average shape by comparing with the ground-truth complete dataset S1. In general, the quality of the registrations allows a very good detection of the missing parts, with the exception of a weak zone situated on the cervical line (not visible in the figures). For S2 (Figure 4), the three cusps analysed are badly represented by the workflow 1 . The workflow 2 allows to improve considerably the shape

\begin{tabular}{|cc|cccc|}
\hline & & All & C1 & C2 & C3 \\
\hline \multirow{2}{*}{ S2 } & $\bar{T}_{1}$ & $0.11(0.21)$ & $0.47(0.18)$ & $0.81(0.38)$ & $0.58(0.23)$ \\
& $\bar{T}_{2}$ & $0.10(0.13)$ & $0.18(0.09)$ & $0.42(0.17)$ & $0.06(0.03)$ \\
\hline \multirow{2}{*}{ S3 } & $\bar{T}_{1}$ & $0.05(0.10)$ & $0.15(0.07)$ & $0.40(0.22)$ & $0.25(0.11)$ \\
& $\bar{T}_{2}$ & $0.05(0.07)$ & $0.15(0.08)$ & $0.24(0.12)$ & $0.05(0.02)$ \\
\hline
\end{tabular}

Table 3: The mean (standard deviation) of the distances (in $\mathrm{mm}$ ) between the average shape of S1 and the average shapes of S2, S3 for the whole surface (All) and for selected areas $(\mathrm{C} 1, \mathrm{C} 2, \mathrm{C} 3)$.

of the cusps. For S3 (Figure 4), we observe the same improvement of the shape of the cusps. The average shape is closer to S1. For S4 (Figure 5), the shape is slightly modified after workflow 2 . This result could be explained by the small number of samples in S4.

To quantify the differences, we compute the mean distances (Table 3 on the whole tooth and on reduced areas including cusps (Figure 3). These distances show that the selection of momenta to compute the deformation improves in a strong way the reconstruction of the average shape for $\mathrm{S} 2$ and $\mathrm{S} 3$. Workflow 2 reduces the mean distances for the three cusps. We observe that $\mathrm{C} 2$ in $\mathrm{S} 2$ is mainly represented by one sample, and there is a lateral displacement of this cusp. Concerning S3, the mean distances for the two workflows are the same for $\mathrm{C} 1$ but these distances are divided by two for $\mathrm{C} 2$ and $\mathrm{C} 3$.

We test the elimination of non-common part on the sample S2. For samples that were strongly damaged, the Figure 6 shows that only equivalent areas are conserved after the cutting. Each sample contains at the end only comparable information and can then be used for a new registration process. 

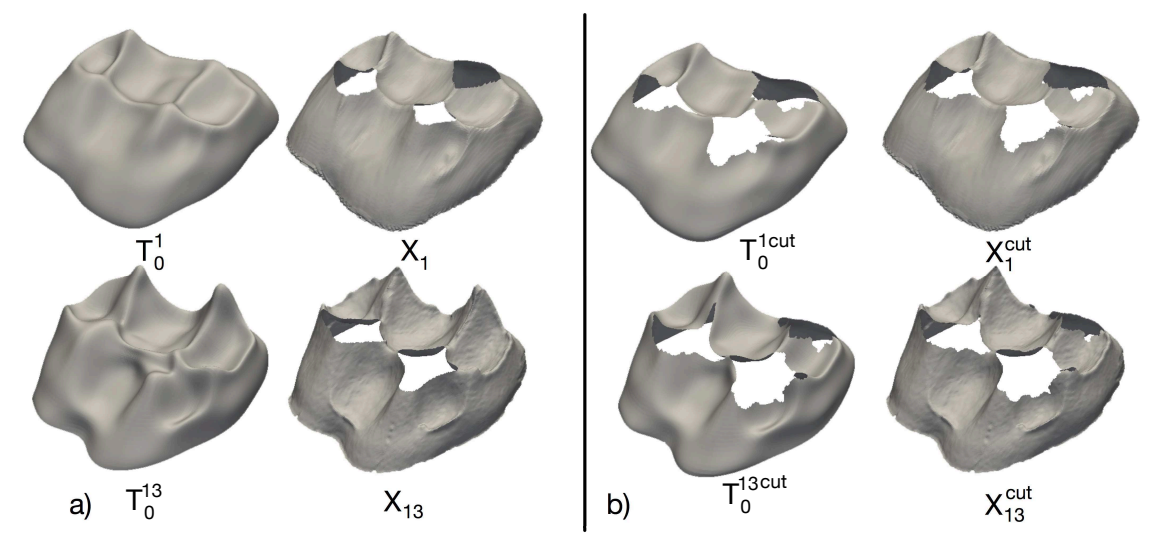

Figure 6: Workflow 3 performed on both the the deformed template and on the sample set of S2. a) original samples, b) cut surfaces.

\section{Conclusion}

In this paper we discussed the problem of estimating an average shape when some samples are incomplete. Furthermore, we proposed a method allowing to not take into account the missing parts of each sample and we show that the registration results could be improved, leading to a better averaging of samples. In future work, we plan to improve the detection of missing parts which can be a true hole (as in our application) or the absence of a characteristic structure (as an anatomical variant). It could be made in particular by applying many iterations of the different presented workflows. We also plan to study the influence of the parameters of the registration algorithm (e.g. the smoothing parameter) in detecting missing parts. Another critical perspective is to test the process on much larger databases to evaluate the performance regarding the statistical analysis of shape for classification. In particular, we will apply our methodology on medical datasets in order to emphasize anatomical variants (as proposed in [15]). In terms of paleontological perspectives, the development of computer-assisted methods for the reconstruction of missing parts will contribute to a better evaluation of the paleobiodiversity by including the numerous incomplete samples that are currently excluded from analyses because of potential bias.

\section{Acknowledgments}

This work was granted access to the HPC resources of CALMIP supercomputing center under the allocation 2016[P1440] attributed to the AMIS laboratory. This Research was supported by the French Ministry of Foreign Affairs and the CNRS (Centre National de la Recherche Scientifique).

\section{References}

[1] M. A. Audette, F. P. Ferrie, and T. M. Peters. An algorithmic overview of surface registration techniques for medical imaging. Medical Image Analysis, 4(3):201 - 217, 2000.

[2] A. Beaudet. Caractérisation des structures crânio-dentaires internes des cercopithécoüdes et étude diachronique de leurs variations morphologiques dans la séquence PlioPléistocène sud-africaine. $\mathrm{PhD}$ thesis, Université de Toulouse, Nov. 2015.

[3] A. Beaudet, J. Dumoncel, J. F. Thackeray, L. Bruxelles, B. Duployer, C. Tenailleau, L. Bam, K. Hoffman, F. de Beer, and J. Braga. Upper third molar internal structural organization and semicircular canal morphology in Plio-Pleistocene South African cercopithecoids. 2016.

[4] S. Benazzi, C. Fornai, L. Buti, M. Toussaint, F. Mallegni, S. Ricci, G. Gruppioni, G. W. Weber, S. Condemi, and A. Ronchitelli. Cervical and crown outline analysis of worn Neanderthal and modern human lower second deciduous molars. American Journal of Physical Anthropology, 149(4):537-546, Oct. 2012.

[5] P. J. Besl and N. D. McKay. Method for registration of 3-D shapes. IEEE Transactions on Pattern Analysis and Machine Intelligence, 14(2):239-256, 1992.

[6] F. L. Bookstein. Morphometric Tools for Landmark Data. Cambridge University Press, 1992. Cambridge Books Online.

[7] D. M. Boyer, Y. Lipman, E. S. Clair, J. Puente, B. A. Patel, T. Funkhouser, J. Jernvall, and I. Daubechies. Algorithms to automatically quantify the geometric similarity of anatomical surfaces. Proceedings of the National Academy of Sciences, 108(45):18221-18226, 2011.

[8] J. Braga, F. Thackeray, G. Subsol, J.-L. Kahn, D. Maret, J. Treil, and A. Beck. The enamel-dentine junction (EDJ) in the post-canine dentition of Australopithecus africanus (Sts52): assessment of intra-individual metameric and antimeric variation. Journal of Anatomy, 216(1):62-79, Jan. 2010 . 
[9] X. Cui, S. Zhang, Y. Zhan, M. Gao, J. Huang, and D. N. Metaxas. MICCAI 2012 International Workshop, chapter 3D Anatomical Shape Atlas Construction Using Mesh Quality Preserved Deformable Models, pages 12-21. Springer Berlin Heidelberg, Berlin, Heidelberg, 2012.

[10] A. Duci, A. J. Yezzi, S. Mitter, and S. Soatto. Computer Vision - ECCV 2002: 7th European Conference on Computer Vision Copenhagen, Denmark, May 28-31, 2002 Proceedings, Part III, chapter Region Matching with Missing Parts, pages 48-62. Springer Berlin Heidelberg, Berlin, Heidelberg, 2002.

[11] S. Durrleman, X. Pennec, A. Trouvé, N. Ayache, and J. Braga. Comparison of the endocranial ontogenies between chimpanzees and bonobos via temporal regression and spatiotemporal registration. Journal of Human Evolution, pages 1-15, Nov. 2011.

[12] S. Durrleman, M. Prastawa, N. Charon, J. R. Korenberg, S. Joshi, G. Gerig, and A. Trouvé. Morphometry of anatomical shape complexes with dense deformations and sparse parameters. NeuroImage, 101:35 - 49, 2014.

[13] S. Durrleman, M. Prastawa, J. R. Korenberg, S. Joshi, A. Trouvé, and G. Gerig. MICCAI 2012: 15th International Conference, chapter Topology Preserving Atlas Construction from Shape Data without Correspondence Using Sparse Parameters, pages 223-230. Springer Berlin Heidelberg, Berlin, Heidelberg, 2012.

[14] A. F. Frangi, W. J. Niessen, D. Rueckert, and J. A. Schnabel. Information Processing in Medical Imaging: 17th International Conference, chapter Automatic 3D ASM Construction via Atlas-Based Landmarking and Volumetric Elastic Registration, pages 78-91. Springer Berlin Heidelberg, Berlin, Heidelberg, 2001.

[15] R. Hanna, H. Barschdorf, T. Klinder, F. M. Weber, M. W. Krueger, O. Dössel, and C. Lorenz. Functional Imaging and Modeling of the Heart: 6th International Conference, FIMH 2011, New York City, NY, USA, May 25-27, 2011. Proceedings, chapter A Hybrid Method for Automatic Anatomical Variant Detection and Segmentation, pages 333-340. Springer Berlin Heidelberg, Berlin, Heidelberg, 2011.

[16] D. G. Kendall. Shape manifolds, procrustean metrics, and complex projective spaces. Bulletin of the London Mathematical Society, 16(2):81-121, 1984.

[17] D. G. Kendall. Shape and shape theory. Wiley series in probability and statistics. Wiley, New York, 1999.

[18] P. Mitteroecker and P. Gunz. Advances in Geometric Morphometrics. Evolutionary Biology, 36(2):235-247, Mar. 2009.

[19] J. T. Richtsmeier, J. M. Cheverud, and S. Lele. Advances in anthropological morphometrics. Annual Review of Anthropology, pages 283-305, 1992.

[20] M. M. Skinner, P. Gunz, B. A. Wood, and J.-J. Hublin. Enamel-dentine junction (EDJ) morphology distinguishes the lower molars of Australopithecus africanus and Paranthropus robustus. Journal of Human Evolution, 55(6):979988, 2008.

[21] D. E. Slice. Landmark coordinates aligned by Procrustes analysis do not lie in Kendall's shape space. Systematic biology, pages 141-149, 2001.
[22] R. E. Strauss, M. N. Atanassov, and J. A. De Oliveira. Evaluation of the principal-component and expectationmaximization methods for estimating missing data in morphometric studies. Journal of Vertebrate Paleontology, 23(2):284-296, 2003.

[23] K. Subramanyan and D. Dean. A procedure to average 3D anatomical structures. Medical image analysis, 4(4):317334, 2000.

[24] G. Subsol, J.-P. Thirion, and N. Ayache. A scheme for automatically building three-dimensional morphometric anatomical atlases: application to a skull atlas. Medical image analysis, 2(1):37-60, 1998.

[25] G. K. Tam, Z.-Q. Cheng, Y.-K. Lai, F. C. Langbein, Y. Liu, D. Marshall, R. R. Martin, X.-F. Sun, and P. L. Rosin. Registration of $3 \mathrm{D}$ point clouds and meshes: a survey from rigid to nonrigid. Visualization and Computer Graphics, IEEE Transactions on, 19(7):1199-1217, 2013.

[26] L. A. Yaroch. Shape analysis using the thin-plate spline: Neanderthal cranial shape as an example. American Journal of Physical Anthropology, 101(S23):43-89, 1996.

[27] Z. Zhang. Iterative point matching for registration of freeform curves and surfaces. International Journal of Computer Vision, 13(2):119-152, 1994.

[28] C. Zollikofer and M. de Leon. Virtual Reconstruction: A Primer in Computer-Assisted Paleontology and Biomedicine. Wiley, 2005. 